\title{
Standard Risk Aversion and Efficient Risk Sharing
}

\author{
Richard M. H. Suen*
}

This Version: 6th September, 2018.

\begin{abstract}
This paper analyzes the risk attitude and investment behavior of a group of heterogeneous consumers who face an uninsurable background risk. It is shown that standard risk aversion at the individual level does not imply standard risk aversion at the group level under efficient risk sharing. This points to a potential divergence between individual and collective portfolio choices in the presence of background risk. We show that if the members' absolute risk tolerance is increasing and satisfies a strong form of concavity, then the group has standard risk aversion.
\end{abstract}

Keywords: Standard risk aversion; Efficient risk sharing; Background risk; Portfolio choice.

JEL classification: D70, D81, G11.

\footnotetext{
${ }^{*}$ School of Business, University of Leicester, University Road, Leicester LE1 7RH, United Kingdom. Phone: +44116252 2880. Email: mhs15@le.ac.uk.
} 


\section{Introduction}

Both conventional wisdom and empirical evidence suggest that people are more reluctant to invest in risky assets when they face other sources of uninsurable and undesirable "background" risk (e.g., labor income risk). ${ }^{1}$ In a seminal paper, Kimball (1993) shows that an expected-utility maximizer with decreasing absolute risk aversion (DARA) and decreasing absolute prudence (DAP) will have this type of response to background risk. The combination of DARA and DAP is referred to as standard risk aversion. In the present study, we ask whether a group of diverse individuals, who share risks efficiently among themselves and make joint investment decisions, will respond to background risk in the same way. Specifically, we want to identify the conditions under which the group's preferences (or aggregate utility function) exhibit standard risk aversion.

It is known that if all members have DARA preferences, then the aggregate utility function will have the same property. ${ }^{2}$ However, this is not true in general for DAP, as we will show below. In other words, standard risk aversion at the individual level is not enough to ensure standard risk aversion at the group level under an efficient risk-sharing arrangement. One implication is that a group of standard-risk-averse individuals under such arrangement may choose to increase their exposure to risky assets in the presence of background risk. A specific example is shown in Section 3. In this paper, we ask the question: under what conditions will collective portfolio choices under background risk be consistent with individual choices? Our main result shows that if each individual member's absolute risk tolerance is increasing and satisfies a strong form of concavity (which implies DAP) then the aggregate utility function is standard. This result has two other implications on the group's preferences. Firstly, since standard risk aversion implies proper risk aversion and risk vulnerability, our result ensures that the group's preferences will have these properties. ${ }^{3}$ Secondly, DAP implies that the aggregate utility function has a negative fourth derivative. ${ }^{4}$ Apps et al. (2014) show that this property is not guaranteed in general even if all the members' utility function have negative fourth derivative.

\footnotetext{
${ }^{1}$ See, for instance, Heaton and Lucas (2000) and Palia et al. (2014) for empirical evidence.

${ }^{2}$ See, for instance, Hara et al. (2007, p.656) for a formal statement of this result.

${ }^{3}$ The notions of "proper risk aversion" and "risk vulnerability" are introduced by Pratt and Zeckhauser (1987) and Gollier and Pratt (1996), respectively.

${ }^{4}$ This property is often referred to as "temperance." See, Kimball (1992) and Eeckhoudt and Schlesinger (2006) for more discussion on this property.
} 


\section{The Model}

Consider a static model with a group made up of $N$ individuals, $N$ being an integer greater than one. The group has a sure amount of initial wealth $W>0$, which can be invested in two assets: a safe asset with a riskless rate of return $r>0$ and a risky asset with a random rate of return $\widetilde{R}$. Let $\alpha$ and $W-\alpha$ denote, respectively, the amount of risky and safe investment. The gross return from this portfolio is given by

$$
(W-\alpha)(1+r)+\alpha(1+\widetilde{R})=\omega+\alpha \widetilde{x}
$$

where $\omega \equiv W(1+r)>0$ and $\widetilde{x} \equiv \widetilde{R}-r$ is the excess return from the risky asset. The random variable $\widetilde{x}$ is drawn from a compact interval $X \subseteq \mathbb{R}$ according to some probability distribution. Apart from the risky investment, the group also faces an exogenous, uninsurable background risk $\widetilde{y}$ in final wealth. The background risk is drawn from a compact interval $Y \subseteq \mathbb{R}$; it can take both positive and negative values and is statistically independent of $\widetilde{x}{ }^{5}$ The probability distributions of $\widetilde{x}$ and $\widetilde{y}$ are known to all group members, so there is no disagreement in their probabilistic beliefs. The sum of investment returns and background risk is used to finance the members' consumption. The group as a whole thus faces the following budget constraint:

$$
\sum_{i=1}^{N} \widetilde{c}_{i} \leq \omega+\alpha \widetilde{x}+\widetilde{y}
$$

where $\widetilde{c}_{i}$ denotes member $i$ 's consumption. Each member's preferences can be represented by $E\left[u_{i}\left(\widetilde{c}_{i}\right)\right]$, for $i \in\{1,2, \ldots, N\}$. The utility function $u_{i}: \mathbb{R}_{+} \rightarrow \mathbb{R}$ is at least five times differentiable, strictly increasing, strictly concave and satisfies the Inada condition $\lim _{c \rightarrow 0} u_{i}^{\prime}(c)=\infty$.

We focus on efficient decisions made by the group. Specifically, the members of the group collectively decide on a level of risky investment $(\alpha)$ and an allocation of consumption $\left(\widetilde{c}_{1}, \widetilde{c}_{2}, \ldots, \widetilde{c}_{N}\right)$

\footnotetext{
${ }^{5}$ One example of such background risk is household earnigs risk. In particular, a positive value of $\widetilde{y}$ can be interpreted as a positive deviation in household earnings from its expected value. This can be the result of a promotion or bonus. On the other hand, a negative value (or negative deviation from the mean) can be the result of a layoff. The independence assumption is commonly used in the background risk literature [e.g., Pratt and Zeckhauser (1987), Kimball (1993) and Gollier and Pratt (1996), among many others]. It is also consistent with the empirical evidence reported in Palia et al. (2014, Table 2). There is a burgeoning literature that considers intrahousehold risk sharing in the presence of uninsurable earnings risk [see, for instance, Mazzocco (2007) and Ortigueira and Siassi (2013)].
} 
so as to maximize a weighted sum of their expected utility, i.e.,

$$
\sum_{i=1}^{N} \lambda_{i} E\left[u_{i}\left(\widetilde{c}_{i}\right)\right]
$$

where $\lambda_{i}>0$ is the Pareto weight for member $i$; subject to (1) and $\widetilde{c}_{i} \geq 0$ for all $i$. This problem can be divided into two parts: First, conditional on $\alpha$ and the realization of $(\widetilde{x}, \widetilde{y})$, the group solves a resources allocation problem:

$$
\widehat{u}(z) \equiv \max _{\left\{\widetilde{c}_{1}, \ldots, \widetilde{c}_{N}\right\}} \sum_{i=1}^{N} \lambda_{i} u_{i}\left(\widetilde{c}_{i}\right)
$$

subject to

$$
\sum_{i=1}^{N} \widetilde{c}_{i} \leq z \equiv \omega+\alpha \widetilde{x}+\widetilde{y}, \quad \text { and } \quad \widetilde{c}_{i} \geq 0 \quad \text { for all } i .
$$

For any $z>0$, the constraint set of the above problem is compact. This, together with a continuous and strictly concave objective function, ensures the existence of a unique solution. The Inada condition ensures that each optimal $\widetilde{c}_{i}$ is strictly positive. By the maximum theorem, the aggregate utility function $\widehat{u}(\cdot)$ is continuous and each optimal $\widetilde{c}_{i}$ can be determined by a continuous function $\kappa_{i}(z)$, known as the sharing rule. By the implicit function theorem, if each $u_{i}(\cdot)$ is $(m+1)$ times differentiable, then both $\kappa_{i}(\cdot)$ and $\widehat{u}(\cdot)$ are $m$ times differentiable. Thus, under our stated assumptions, both $\kappa_{i}(\cdot)$ and $\widehat{u}(\cdot)$ are at least four times differentiable. In addition, $\widehat{u}(\cdot)$ is strictly increasing and strictly concave.

The second part of the group problem is to choose the level of risky investment, i.e.,

$$
\max _{\alpha} E[\widehat{u}(\omega+\alpha \widetilde{x}+\widetilde{y})]
$$

Since the optimal choice of all $\widetilde{c}_{i}$ must be strictly positive, the group must choose $\alpha$ so that $z \equiv \omega+\alpha \widetilde{x}+\widetilde{y}$ is strictly positive for all possible realizations of $(\widetilde{x}, \widetilde{y})$. Depending on the boundary values of $X$ and $Y$, this can allow for short-selling of the risky asset (i.e., $\alpha<0$ ) or short-selling of the safe asset (i.e., $\alpha>W$ ). Since the objective function in (3) is continuous and strictly concave in $\alpha$, a unique solution (denoted by $\alpha^{*}$ ) exists. 


\section{Standard Risk Aversion of $\widehat{u}$}

For each member $i \in\{1,2, \ldots, N\}$, define $A_{i}(c) \equiv-u_{i}^{\prime \prime}(c) / u_{i}^{\prime}(c)$ as the measure of absolute risk aversion and $P_{i}(c) \equiv-u_{i}^{\prime \prime \prime}(c) / u_{i}^{\prime \prime}(c)$ as the measure of absolute prudence. The reciprocal of $A_{i}(c)$, denoted by $T_{i}(c)$, is the measure of absolute risk tolerance. The first derivative of $T_{i}(c)$ is referred to as absolute cautiousness [see Wilson (1968)]. Since $\widehat{u}(\cdot)$ is at least four times differentiable, we can define the corresponding measures, $\widehat{A}(z), \widehat{T}(z)$ and $\widehat{P}(z)$, for the aggregate utility function. Wilson (1968) shows that there is a close connection between $T_{i}(c)$, $\widehat{T}(z)$ and $\kappa_{i}(z)$. Specifically,

$$
\begin{gathered}
\kappa_{i}^{\prime}(z)=\frac{T_{i}\left[\kappa_{i}(z)\right]}{\widehat{T}(z)}>0 \quad \text { for all } i, \text { and } \\
\widehat{T}(z)=\sum_{i=1}^{N} T_{i}\left[\kappa_{i}(z)\right] .
\end{gathered}
$$

Differentiating both sides of (5) with respect to $z$ gives

$$
\widehat{T}^{\prime}(z)=\sum_{i=1}^{N} \kappa_{i}^{\prime}(z) T_{i}^{\prime}\left[\kappa_{i}(z)\right]
$$

Since $\sum_{i=1}^{N} \kappa_{i}^{\prime}(z)=1$, the absolute cautiousness of $\widehat{u}(\cdot)$ can be viewed as a weighted average of the individuals' absolute cautiousness (evaluated under the sharing rule).

We now consider the effect of background risk on the group's investment decision. Note that the portfolio choice problem in (3) is no different from the one faced by a single decision-maker (normative representative agent) with utility function $\widehat{u}(\cdot)$. Thus, according to the variant of Proposition 6 in Kimball (1993, p.610), any independent background risk $\widetilde{y}$ that raises the representative agent's expected marginal utility under the optimal choice $\alpha^{*}$, i.e.,

$$
E\left[\widehat{u}^{\prime}\left(\omega+\alpha^{*} \widetilde{x}+\widetilde{y}\right)\right] \geq E\left[\widehat{u}^{\prime}\left(\omega+\alpha^{*} \widetilde{x}\right)\right]
$$

will lower the absolute value of $\alpha^{*}$ if and only if $\widehat{u}(\cdot)$ exhibits standard risk aversion, i.e., when both $\widehat{A}(\cdot)$ and $\widehat{P}(\cdot)$ are decreasing functions.

From (4) and (5), it is obvious that if $T_{i}(\cdot)$ is an increasing function (or equivalently, $A_{i}(\cdot)$ is a decreasing function) for all $i$, then $\widehat{A}(\cdot)$ must be decreasing. The relation between $P_{i}(\cdot)$ and $\widehat{P}(\cdot)$ is examined in Lemma 1 . 
Lemma 1 The representative agent's absolute prudence is given by

$$
\widehat{P}(z) \equiv \sum_{i=1}^{N}\left[\kappa_{i}^{\prime}(z)\right]^{2} P_{i}\left[\kappa_{i}(z)\right]
$$

with first derivative

$$
\widehat{P}^{\prime}(z)=\sum_{i=1}^{N}\left[\kappa_{i}^{\prime}(z)\right]^{3} P_{i}^{\prime}\left[\kappa_{i}(z)\right]+\frac{2}{[\widehat{T}(z)]^{2}} \sum_{i=1}^{N} \kappa_{i}^{\prime}(z)\left\{T_{i}^{\prime}\left[\kappa_{i}(z)\right]-\widehat{T}^{\prime}(z)\right\}^{2}
$$

Proof of Lemma 1 Differentiating $T_{i}(c) \equiv-u_{i}^{\prime}(c) / u_{i}^{\prime \prime}(c)$ with respect to $c$ gives $T_{i}^{\prime}(c)=$ $-1+T_{i}(c) P_{i}(c)$ for all $c>0$. The counterpart for $\widehat{u}(\cdot)$ is $\widehat{T}^{\prime}(z)=-1+\widehat{T}(z) \widehat{P}(z)$ for all $z>0$. Substituting these questions into (6), and using $\sum_{i=1}^{N} \kappa_{i}^{\prime}(z)=1$ gives

$$
\widehat{T}(z) \widehat{P}(z)=\sum_{i=1}^{N} \kappa_{i}^{\prime}(z) T_{i}\left[\kappa_{i}(z)\right] P_{i}\left[\kappa_{i}(z)\right]
$$

Equation (8) follows immediately by rearranging terms and applying (4). Next, differentiating (8) with respect to $z$ gives

$$
\widehat{P}^{\prime}(z)=\sum_{i=1}^{N}\left[\kappa_{i}^{\prime}(z)\right]^{3} P_{i}^{\prime}\left[\kappa_{i}(z)\right]+2 \sum_{i=1}^{N} \kappa_{i}^{\prime}(z) \kappa_{i}^{\prime \prime}(z) P_{i}\left[\kappa_{i}(z)\right]
$$

Differentiating (4) with respect to $z$ gives

$$
\kappa_{i}^{\prime \prime}(z)=\frac{\kappa_{i}^{\prime}(z)}{\widehat{T}(z)}\left\{T_{i}^{\prime}\left[\kappa_{i}(z)\right]-\widehat{T}^{\prime}(z)\right\}
$$

Equation (9) can be obtained by combining the last two equations.

Equation (9) shows that the first derivative of $\widehat{P}(\cdot)$ can be decomposed into two parts: The first part captures the effects of $P_{i}^{\prime}(\cdot)$ on $\widehat{P}^{\prime}(\cdot)$. In particular, this term is negative if all group members have decreasing absolute prudence. The second term captures the effects due to the heterogeneity in absolute cautiousness across group members. Since $\widehat{T}^{\prime}(z)$ is the weighted average of $\left\{T_{i}^{\prime}\left[\kappa_{i}(z)\right]\right\}_{i=1}^{N}$ under the set of weights $\left\{\kappa_{i}^{\prime}(z)\right\}_{i=1}^{N}$, the expression $\sum_{i=1}^{N} \kappa_{i}^{\prime}(z)\left\{T_{i}^{\prime}\left[\kappa_{i}(z)\right]-\widehat{T}^{\prime}(z)\right\}^{2}$ is the variance of absolute cautiousness among the group members, which is always positive. Thus, even if all members have DAP preferences, the representative agent may not. In this case, it is possible to find $\{\widetilde{x}, \widetilde{y}\}$ such that the group will increase 


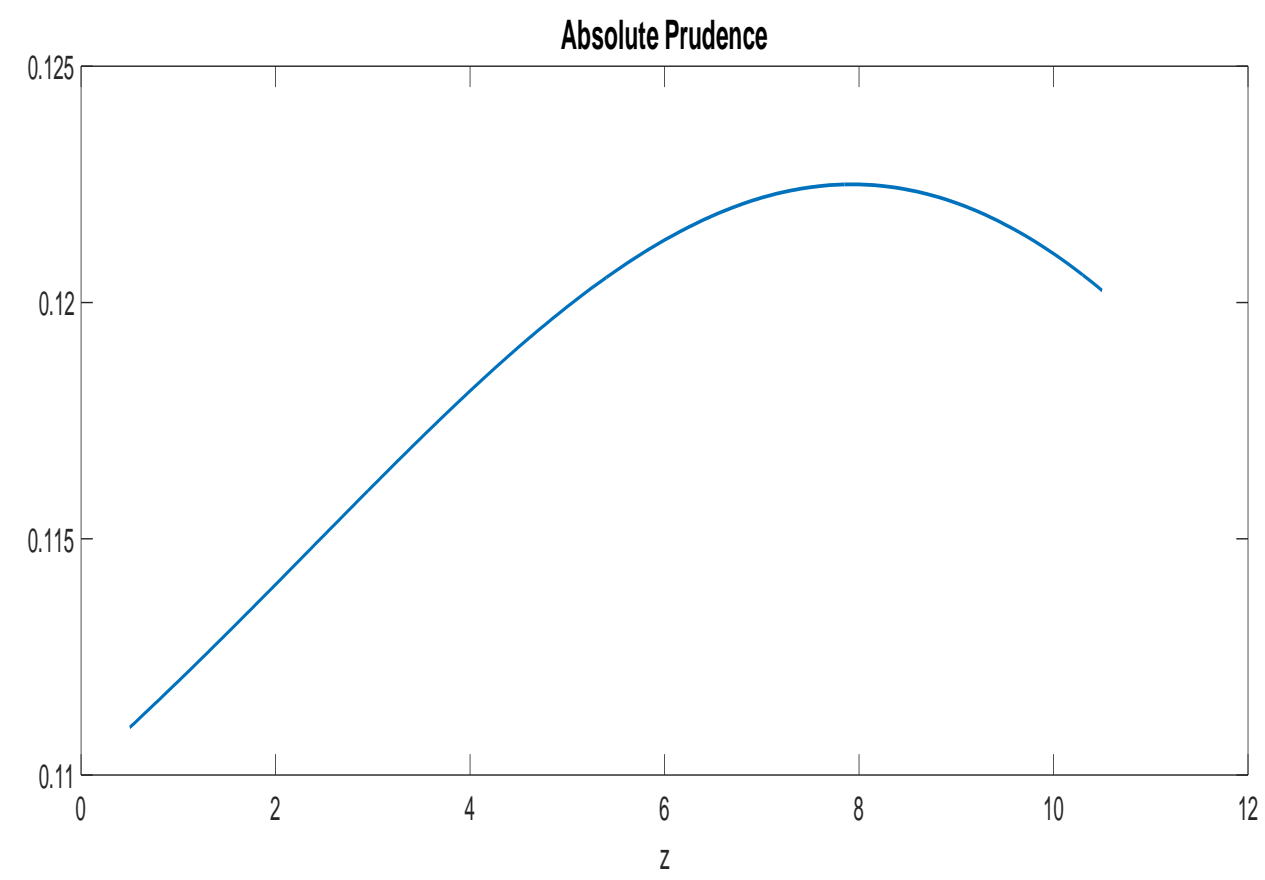

Figure 1: An Example of Non-monotonic $\widehat{P}(\cdot)$.

their risky investment in the presence of background risk. This is demonstrated in the following example.

Consider two individuals with preferences $u_{1}(c)=1-\phi^{-1} \exp (-\phi c)$ and $u_{2}(c)=\left(c^{1-\sigma}-1\right) /(1-\sigma)$, with $\phi>0$ and $\sigma>0$. Both individuals exhibit (weakly) decreasing absolute risk aversion and (weakly) decreasing absolute prudence. When acting alone, the first agent's choice of $\alpha$ is unaffected by any background risk that satisfies (7), while the second agent will reduce his/her risky investment. Suppose now the two form an efficient risk-sharing group and suppose $\lambda_{1}=1.5$, $\lambda_{2}=1.0, \phi=0.1$ and $\sigma=0.4$. The resulting $\widehat{P}(z)$, as depicted in Figure 1, is non-monotonic and strictly increasing when $z$ is small. Take $\omega=4.5$ and suppose $\widetilde{x}$ has only two possible states, -0.2 and 0.24 , with equal probability. In the absence of any background risk, the couple's optimal choice of risky investment is $\alpha_{1}^{*}=5.093$. Suppose now we introduce a background risk $\widetilde{y}$, which can take three possible values: $-2.0,0$ and 2.6 , with equal probability. ${ }^{6}$ In the presence of $\widetilde{y}$, the couple will increase their risky investment to $\alpha_{2}^{*}=5.105$.

The results in our Lemma 1 are closely related to those in Hara et al. (2007, Section 4). Specifically, these authors show that efficient risk sharing has a tendency to make $\widehat{T}(z)$ a

\footnotetext{
${ }^{6}$ Condition (7) is satisfied under this $\widetilde{y}$ and $\alpha_{1}^{*}$. The detail of this and other parts of the example are shown in a Technical Appendix available from the author's personal website. In this appendix, we show that similar results [i.e., a non-monotonic $\widehat{P}(\cdot)$ and $\alpha_{2}^{*}>\alpha_{1}^{*}$ ] can be obtained under other values of $\left\{\lambda_{1}, \sigma, \phi\right\}$.
} 
convex function and increase the slope of $\widehat{T}(z) / z$. Thus, even if all group members have concave absolute risk tolerance or increasing relative risk aversion (which is equivalent to a decreasing $\left.T_{i}(c) / c\right)$, the representative agent may not have these characteristics. The concavity of $\widehat{T}(\cdot)$ is of particular interest here due to the following observation. ${ }^{7}$

Lemma 2 If $\widehat{T}(\cdot)$ is increasing concave, then $\widehat{P}(\cdot)$ is decreasing and $\widehat{u}(\cdot)$ is standard.

Lemma 2 suggests one way to establish the standardness of $\widehat{u}(\cdot)$. The remaining question is under what conditions will $\widehat{T}(\cdot)$ be a concave function. Hara et al. (2007) have already shown that it is not enough to have a concave $T_{i}(\cdot)$ for all $i$. This prompts us to consider a stronger form of concavity, which is the notion of " $\rho$-concavity" as discussed in Caplin and Nalebuff (1991).

For any $\rho \in[-\infty, \infty]$, a nonnegative function $g(\cdot)$ is called $\rho$-concave if the transformed function $\widetilde{g}(x) \equiv[g(x)]^{\rho} / \rho$ is concave. Since $g(\cdot)$ and $\widetilde{g}(\cdot)$ are equivalent when $\rho=1$, the usual notion of concavity corresponds to the case of $\rho=1 .^{8}$ In general, if $g(\cdot)$ is $\rho_{1}$-concave, then it is also $\rho_{2}$-concave for all $\rho_{2} \leq \rho_{1}$. If both $g(\cdot)$ and $\widetilde{g}(\cdot)$ are twice differentiable, then $g(\cdot)$ is $\rho$-concave if and only if

$$
g(x) g^{\prime \prime}(x) \leq(1-\rho)\left[g^{\prime}(x)\right]^{2}, \quad \text { for all } x
$$

The main result of this paper is to show that if each group member's absolute risk tolerance is $\rho$-concave, for some $\rho \geq 2$, then the representative agent's absolute risk tolerance is a concave function. This result holds regardless of whether $T_{i}(\cdot)$ is monotonic. It follows that if each $T_{i}(\cdot)$ is increasing and $\rho$-concave, for some $\rho \geq 2$, then $\widehat{T}(\cdot)$ is increasing concave and $\widehat{u}(\cdot)$ is standard..$^{9}$

Theorem 3 Suppose for each $i \in\{1,2, \ldots, N\}, T_{i}(\cdot)$ is $\rho$-concave, for some $\rho \geq 2$, then $\widehat{T}(\cdot)$ is a concave function. If, in addition, each $T_{i}(\cdot)$ is increasing, then $\widehat{u}(\cdot)$ is standard.

Proof of Theorem 3 As shown in Theorem 4 of Hara et al. (2007), the second derivative of $\widehat{T}(z)$ can be expressed as

$$
\widehat{T}^{\prime \prime}(z)=\sum_{i=1}^{N}\left[\kappa_{i}^{\prime}(z)\right]^{2} T_{i}^{\prime \prime}\left[\kappa_{i}(z)\right]+\frac{1}{\widehat{T}(z)} \sum_{i=1}^{N} \kappa_{i}^{\prime}(z) T_{i}^{\prime}\left[\kappa_{i}(z)\right]\left\{T_{i}^{\prime}\left[\kappa_{i}(z)\right]-\widehat{T}^{\prime}(z)\right\} .
$$

\footnotetext{
${ }^{7}$ This result has appeared in Gollier (2001b, p.166). Its proof follows immediately by noting that $\widehat{P}(z)>0$, $\widehat{T}(z)>0$ and $\widehat{T}^{\prime \prime}(z)=\widehat{T}^{\prime}(z) \widehat{P}(z)+\widehat{T}(z) \widehat{P}^{\prime}(z)$ for all $z>0$.

${ }^{8}$ Quasi-concavity and logconcavity of $g(\cdot)$ correspond, respectively, to the cases of $\rho=-\infty$ and $\rho=0$.

${ }^{9}$ If $T_{i}(\cdot)$ is increasing and $\rho$-concave for some $\rho \geq 2$, then it is increasing and concave in the usual sense. Thus, by Lemma $2, u_{i}(\cdot)$ has standard risk aversion.
} 
Using (4) and (6), we can rewrite this as

$$
\widehat{T}^{\prime \prime}(z)=\frac{1}{\widehat{T}(z)} \sum_{i=1}^{N}\left[\kappa_{i}^{\prime}(z)\right]\left\{T_{i}^{\prime \prime}\left[\kappa_{i}(z)\right] T_{i}\left[\kappa_{i}(z)\right]+\left(T_{i}^{\prime}\left[\kappa_{i}(z)\right]\right)^{2}\right\}-\frac{\left[\widehat{T}^{\prime}(z)\right]^{2}}{\widehat{T}(z)} .
$$

Thus, it suffice to show that $T_{i}^{\prime \prime}(c) T_{i}(c)+\left[T_{i}^{\prime}(c)\right]^{2} \leq 0$ for all $c \geq 0$ and for all $i$. If $T_{i}(\cdot)$ is $\rho$-concave for some $\rho \geq 2$, then we have $T_{i}^{\prime \prime}(c) T_{i}(c) \leq(1-\rho)\left[T_{i}^{\prime}(c)\right]^{2}$, which implies

$$
T_{i}^{\prime \prime}(c) T_{i}(c)+\left[T_{i}^{\prime}(c)\right]^{2} \leq(2-\rho)\left[T_{i}^{\prime}(c)\right]^{2} \leq 0
$$

This completes the proof.

In the economics literature, the assumption of $\rho$-concavity is typically imposed on the density function of some distributions. ${ }^{10}$ To the best of our knowledge, this is the first study that applies this type of concavity to characterize risk preferences. Suppose individuals' absolute risk tolerance takes a power form as in Gollier (2001a, p.189), i.e., $T_{i}(c)=\alpha_{i} c^{\phi_{i}}$, for some constants $\alpha_{i}>0$ and $\phi_{i} \geq 0$. Then $T_{i}(c)$ is $\rho$-concave for some $\rho \geq 2$ if and only if $\phi_{i} \leq 0.5$.

\footnotetext{
${ }^{10}$ For instance, Caplin and Nalebuff (1991) impose this assumption on the distribution of voters' characteristics in a voting model; Ewerhart (2013) applies this on the distribution of bidders' characteristics in auction models.
} 


\section{References}

[1] Apps, P., Andrienko, Y., Rees, R., 2014. Risk and precautionary saving in two-person households. American Economic Review, 104, 1040-1046.

[2] Caplin, A., Nalebuff, B., 1991. Aggregation and social choice: a mean voter theorem. Econometrica, 59, 1-23.

[3] Eeckhoudt, L., Schlesinger, H., 2006. Putting risk in its proper place. American Economic Review, 96, 280-289.

[4] Ewerhart, C., 2013. Regular type distributions in mechanism design and $\rho$-concavity. Economic Theory, 53, 591-603.

[5] Gollier, C. 2001a. Wealth inequality and asset pricing. Review of Economic Studies, 68, 181-203.

[6] Gollier, C. 2001b. The economics of risk and time. The MIT Press, Cambridge, MA.

[7] Gollier, C., Pratt, J.W., 1996. Risk vulnerability and the tempering effect of background risk. Econometrica, 64, 1109-1123.

[8] Hara, C., Huang, J., Kuzmics, C., 2007. Representative consumer's risk aversion and efficient risk-sharing rules. Journal of Economic Theory, 137, 652-672.

[9] Heaton, J., Lucas, D., 2000. Portfolio choice in the presence of background risk. Economic Journal, 110, 1-26.

[10] Kimball, M.S., 1992. Precautionary motives for holding assets, in:. Newman, P., Milgate, M., Falwell, J., (Ed.) The New Palgrave Dictionary of Money and Finance, MacMillan, London, 158-161.

[11] Kimball, M.S., 1993. Standard risk aversion. Econometrica, 61, 589-611.

[12] Mazzocco, M., 2007. Household intertemporal behaviour: a collective characterization and a test of commitment. Review of Economic Studies, 74, 857-895.

[13] Ortigueira, S., Siassi, N., 2013. How important is intra-household risk sharing for savings and labor supply? Journal of Monetary Economics, 60, 650-666. 
[14] Palia, D., Qi, Y., Wu, Y., 2014. Heterogeneous background risks and portfolio choice: evidence from micro-level data. Journal of Money, Credit and Banking, 46, 1687-1720.

[15] Pratt, J.W., Zeckhauser, R.J., 1987. Proper risk aversion. Econometrica, 55, 143-154.

[16] Wilson, R., 1968. The theory of syndicates. Econometrica, 36, 119-132. 